\title{
Fairness and utilitarianism without independence
}

\author{
Sinong Ma ${ }^{1} \cdot$ Zvi Safra $^{2,3}{ }_{\mathbb{D}}$
}

Received: 29 March 2017 / Accepted: 30 November 2017 / Published online: 12 December 2017 (C) The Author(s) 2017. This article is an open access publication

\begin{abstract}
In this work we reconsider Harsanyi's celebrated (J Polit Econ 61:434-435, 1953; J Polit Econ 63:309-321, 1955; Rational Behavior and Bargaining Equilibrium in Games and Social Situations. Cambridge University Press, Cambridge, 1977) utilitarian impartial observer theorem. Departing from Harsanyi's individual-centered approach, we argue that when societal decisions are at stake, postulates must not be drawn from individualistic behavior. Rather, they should be based on societal norms. Continuing this line of thinking, we state and prove a utilitarian result that, rather than being based on the independence assumption, is based on the societal norm of procedural fairness.
\end{abstract}

Keywords Fairness · Utilitarianism · Impartial observer · Social welfare function

\section{JEL Classification D63 · D71}

\begin{abstract}
"An axiomatic justification of utilitarianism would have more content to it if it started off at a place somewhat more distant from the ultimate destination"
\end{abstract}

(Sen 1976, page 251)

\footnotetext{
We are grateful to David Dillenberger, Simon Grant, Edi Karni, Tigran Melkonyan, Uzi Segal, Jean-Marc Tallon, two anonymous referees and, especially, to Marcus Pivato for very useful comments and suggestions.

The order of authors names was determined by a toss of a fair coin.
}

\section{Zvi Safra}

Zvi.Safra@wbs.ac.uk

1 Warwick Manufacturing Group, University of Warwick, Coventry CV4 7AL, UK

2 Warwick Business School, University of Warwick, Coventry CV4 7AL, UK

3 Emeritus professor, Tel Aviv University, Tel Aviv, Israel 


\section{Introduction}

In this work we reconsider Harsanyi's celebrated $(1953,1955,1977)$ utilitarian impartial observer theorem. We propose an approach that puts more emphasis on procedural fairness, and we offer a utilitarian result that does not use the independence assumption.

Harsanyi analyzed a society that needs to choose among alternate social policies, each of which is a probability distribution over a given set of social actions, where the latter associate outcomes with the society's members. Every social lottery $\ell$ induces a lottery $\ell_{i}$ on individual $i$. Individual $i$ 's preferences $\succcurlyeq_{i}$ are known, and different individuals may possess distinct preferences.

To help determine the optimal social policy, Harsanyi suggested that every individual is endowed with social preferences. Individuals may develop these preferences by adopting the role of an impartial observer, thus disregarding their true identities and acting behind "a veil of ignorance". Therefore, the impartial observer can form her social preferences by imagining that she faces not only a lottery $\ell$ over social actions, but also a lottery $\gamma$ over identities. Elements of $\gamma$ can be interpreted as weights associated by the impartial observer with the different individuals. Then, the optimal social policy is determined by restricting attention to the equiprobable lottery $\gamma^{e}=\left(\frac{1}{n}, \ldots, \frac{1}{n}\right)$.

Harsanyi argued strongly for "Bayesian rationality". That is, he assumed that (among the other Bayesian postulates) all individuals satisfy the independence assumption of the expected utility theory, both at their personal and social preference layers. Harsanyi claimed that this "sound" axiom, together with the so-called acceptance principle (that an impartial observer fully adopts individual $i$ 's preferences if she imagines becoming that individual for sure), would force the impartial observer to be a (weighted) utilitarian. More formally, over all extended lotteries $(\gamma, \ell)$ in which the identity and the action lotteries are independently distributed, the impartial observer's preferences admit the following representation:

$$
V(\gamma, \ell)=\sum_{i \in \mathscr{I}} \gamma_{i} U_{i}\left(\ell_{i}\right)
$$

where $\gamma_{i}$ is the probability of assuming person $i$ 's identity and $U_{i}\left(\ell_{i}\right):=\sum_{x} u_{i}(x) \ell_{i}(x)$ is person $i$ 's von Neumann-Morgenstern expected utility.

Like Harsanyi, most authors who derived modifications of the utilitarianism result within the impartial observer framework always assumed the independence axiom [see the works of Weymark (1991), Karni (1998) and Grant et al. (2010); henceforth, GKPS)]. ${ }^{1}$ Notable exceptions within the related social aggregation framework are Blackorby et al. (2004) and Mongin and Pivato (2015). ${ }^{2}$

Interestingly, Harsanyi's entire emphasis on Bayesian rationality was based on an individual-centered approach. Firstly, he assumed that rational individuals must satisfy the independence assumption and secondly, he claimed that society, by its need to be at least as rational as its members, must also satisfy independence (Harsanyi 1975). We

\footnotetext{
1 A similar observation holds for most of the literature dealing with Harsanyi's social aggregation theorem. See Zhou (1997), Dhillon and Mertens (1999), Gilboa et al. (2004) and Fleurbaey and Mongin (2016).

2 Unlike the other works (including the current one), these authors consider both ex post and ex ante analyses (and thus are able to employ Gorman (1968) separability theorem).
} 
disagree with Harsanyi on this. Instead we argue that when societal decision problems are at stake, postulates must not be drawn from individualistic behavior. Rather, they should be based on societal norms. Hence, when social preferences are formed, issues like societal fairness and equity should explicitly be the guiding principles.

In this work we focus on procedural fairness. This principle was first advocated by Diamond (1967) and was strongly supported by Sen (e.g., 1977). Its essence can be illustrated by the following example, which is an adoption of Diamond's example from the social aggregation framework to the impartial observer one. Consider a society that needs to decide on how to allocate an indivisible good between two individuals, 1 and 2 , and let action $a^{i}$ denote allocating it to individual $i$. Suppose, as Diamond did, that $u_{i}\left(a^{i}\right)=1$ for both $i$ and $u_{i}\left(a^{j}\right)=0$ for $i \neq j$ (that is, both individuals like the good, receive a utility of one unit from having it and zero otherwise). As was noted above assume that, when making a choice, the impartial observer considers the equiprobable identity lottery $\gamma^{e}=\left(\frac{1}{2}, \frac{1}{2}\right)$ (that is, she gives equal weights to the two individuals). Also assume that she evaluates all four outcomes in full agreement with the two individuals and adopts their utilities. The example can be described by the following matrix

\begin{tabular}{c||cc} 
& $a^{1}$ & $a^{2}$ \\
\hline \hline 1 & 1 & 0 \\
2 & 0 & 1
\end{tabular}

where individuals 1 and 2 correspond to the rows, actions $a^{1}$ and $a^{2}$ correspond to the columns and the entries represent the impartial observer's utilities. The impartial observer has two policies at hand: Policy (1), which allocates the good to individual 1 (this policy is equivalent to choosing action $a^{1}$ and facing the first column of the table) and Policy (2), which allocates the good to one of the individuals, depending on the outcome of a toss of a fair coin (this policy is equivalent to the action lottery $\frac{1}{2} a^{1}+\frac{1}{2} a^{2}$ ). The value of Policy (1) for Harsanyi's utilitarian observer is $\frac{1}{2} \times 1+\frac{1}{2} \times 0=\frac{1}{2}$, as is the value of Policy (2): $\frac{1}{2}\left(\frac{1}{2} \times 1+\frac{1}{2} \times 0\right)+\frac{1}{2}\left(\frac{1}{2} \times 0+\frac{1}{2} \times 1\right)=\frac{1}{2}$. Hence, the impartial observer is indifferent between the two policies. ${ }^{3}$ However, Diamond and Sen argued that Policy (2) provides both individuals with a "fair shake" and hence the impartial observer might prefer it. ${ }^{4}$ This notion of procedural fairness is expressed in our work by the notion of (weak) convexity over action lotteries: if, given the identity equiprobable lottery $\gamma^{e}$, the observer is indifferent between two action lotteries $\ell$ and $\ell^{\prime}$ (while two individuals disagree on their ranking) then their mixtures cannot be worse than them. ${ }^{5}$

Working in a framework in which the basic building blocks are two different types of lotteries, those over identities and those over actions, raises a natural question: should these types be treated similarly? Harsanyi, by construction, implicitly assumed

\footnotetext{
3 Note that the impartial observer is also indifferent between $a^{1}$ and $a^{2}$.

4 A long list of real-life applications supporting Diamond's fairness consideration is provided by Elster (1989).

5 Unlike Epstein and Segal (1992), we do not assume that such mixtures are always strictly preferred. This is in agreement with Sen (1977), who argued that mixtures are not always superior.
} 
that they should. Furthermore, in his own response to Diamond's concern about fairness, Harsanyi (1975) argued that even if randomizations were of value for promoting fairness (which he doubted), any explicit randomization is superfluous since "the great lottery of (pre-)life" may be viewed as having already given each child an equal chance of being each individual. That is, it does not matter whether a good is allocated by a (possibly imaginary) lottery over identities or by a (real) lottery over actions. Put it differently, Harsanyi argued that we need to be indifferent between "accidents of birth" (identity lotteries) and real "life chances" (action lotteries). On this issue we agree with Harsanyi and just make this assumption explicit. We call it source indifference.

Despite its innocuous appearance, the conjunction of this assumption with procedural fairness turns out to be rather forceful. More precisely, the main result of this work shows that (assuming impartiality) convexity, source indifference and a stronger notion of acceptance are necessary, and sufficient, for utilitarianism.

Since the independence axiom is not assumed here, this result is novel and quite unexpected. Paraphrasing Sen's quote, we believe that one could hardly find an axiomatic justification of utilitarianism that starts off at a place that is more distant from the ultimate destination than ours.

Lastly, our result carries an 'impossibility flavor': if societies are required to exhibit strict inclination toward procedural fairness, then source indifference cannot hold. Therefore, to accommodate views of authors like Diamond and Sen, the impartial observer must display preference for action lotteries over identity ones. We elaborate on this in the concluding section.

This work is organized as follows: Sect. 2 sets up the framework, Sect. 3 presents the assumptions, Sect. 4 states and explains the utilitarian result, and Sect. 5 concludes. Finally, proofs are given in Sect. 6.

\section{Setup and notation}

Let $\mathscr{X}=\left[x_{\min }, x_{\max }\right] \subset \mathbb{R}$ be a compact interval representing all possible outcomes. ${ }^{6}$ Let $\triangle(\mathscr{X})$ denote the set of outcome lotteries, endowed with the weak convergence topology. With slight abuse of notation, we will let $x$ denote the degenerate outcome lottery that assigns probability 1 to outcome $x$. Let $T$ be a denumerable set of potential individual types, where each type $t \in T$ is characterized by a preference relation over $\triangle(\mathscr{X})$ that is complete, transitive, continuous (in that the weak upper and lower contour sets are closed), increases with respect to first-order stochastic-dominance and its asymmetric part is non-empty. ${ }^{7}$ The set of individuals under consideration is $\mathscr{I}=\cup_{t \in T} \mathscr{I}_{t}$, where $\mathscr{I}_{t}$ is a denumerable (infinite) set of type $t$ individuals. In the sequel, individuals are denoted by $i, j$ (and their preferences by $\succcurlyeq_{i}, \succcurlyeq_{j}$ ), without explicitly specifying their types. A society $I$ is a finite subset of $\mathscr{I}$. Note that, even though we allow for societies in which some individuals are of the same type,

\footnotetext{
6 The end points of the interval, $x_{\min }$ and $x_{\max }$, represent very extreme outcomes. That is, $x_{\max }$ is larger, and $x_{\min }$ is smaller, than any conceivable outcome. For example, $x_{\max }$ can be an outcome that is greater than the combined wealth of any possible society, while $x_{\min }$ can represent a debt of the same extreme size.

7 Note that, by construction, individuals are not themselves sensitive to others' allocated outcomes.
} 
these individuals may receive different outcomes and hence they need not be treated similarly. Also note that our framework departs from Harsanyi's in that, instead of working with one fixed finite society, we consider all finite subsets of $\mathscr{I}{ }^{8}$

A social policy, or an action, associates an outcome with every individual and hence is represented by a function $a: \mathscr{I} \rightarrow \mathscr{X}$. The set of all actions, endowed with the corresponding product topology, is denoted by $\mathscr{A}$ (two extreme actions, $a_{\max }$ and $a_{\min }$, defined by $a_{\max }(i)=x_{\max }$ and $a_{\min }(i)=x_{\min }$ for all $i$, respectively, will be used in the sequel). Let $\triangle(\mathscr{A})$ denote the set of simple lotteries (lotteries with finite support) over actions, with typical elements denoted by $\ell$. With slight abuse of notation, we will let $a$ denote the degenerate action lottery that assigns probability 1 to action $a$. A lottery $\ell \in \triangle(\mathscr{A})$ is sometimes written as $\ell=\sum_{a \in \operatorname{Supp}(\ell)} \ell(a) a$.

Following Harsanyi, an observer imagines herself behind a veil of ignorance, uncertain about which identity she will assume in the given society. Let $\Delta(\mathscr{I})$ denote the set of simple identity lotteries on $\mathscr{I}$, where typical elements are denoted by $\gamma$ (where $\gamma_{i}$ is the probability assigned by the identity lottery $\gamma$ to individual $i$ ). These lotteries represent the imaginary risks in the mind of the observer of being born as someone else. With slight abuse of notation, we will let $i$ denote the degenerate identity lottery that assigns probability 1 to individual $i$. An imaginary lottery $\gamma \in \Delta(\mathscr{I})$ is sometimes written as $\gamma=\sum_{i \in \operatorname{Supp}(\gamma)} \gamma_{i} i$. When the observer is faced with pairs of identity and action lotteries, it is assumed that they are independently distributed.

The observer is endowed with a preference relation $\succcurlyeq$ defined over the space of all product lotteries $\triangle(\mathscr{I}) \times \triangle(\mathscr{A})$. We assume throughout that $\succcurlyeq$ is complete, transitive, continuous and that its asymmetric part $\succ$ is non-empty. These assumptions imply that $\succcurlyeq$ admits a (non-trivial) continuous representation $V: \triangle(\mathscr{I}) \times \Delta(\mathscr{A}) \rightarrow \mathbb{R}$. That is, for any pair of product lotteries $(\gamma, \ell)$ and $\left(\gamma^{\prime}, \ell^{\prime}\right),(\gamma, \ell) \succcurlyeq\left(\gamma^{\prime}, \ell^{\prime}\right)$ if and only if $V(\gamma, \ell) \geqslant V\left(\gamma^{\prime}, \ell^{\prime}\right)$. Note that the observer might not be indifferent between getting some amount $x$ under two different identities (this may happen, for example, if she values affirmative action policies). As a result, there exists no objective natural order over the set of basic identity-outcome pairs $(i, x)$ and, therefore, monotonicity with respect to first-order stochastic-dominance relative to outcomes cannot be assumed. Instead, we require a weaker notion of monotonicity, based on the observer's subjective ranking over $\mathscr{I} \times \mathscr{X}(\operatorname{Im} V$ stands for the image of $V)$

Definition 1 Monotonicity: For any pair of product lotteries $(\gamma, a)$ and $\left(\gamma^{\prime}, a^{\prime}\right)$,

$$
\sum_{\{i: V(i, a) \leqslant v\}} \gamma(i) \leqslant \sum_{\left\{i: V\left(i, a^{\prime}\right) \leqslant v\right\}} \gamma^{\prime}(i) \text { for all } v \in \operatorname{Im} V \Rightarrow(\gamma, a) \succcurlyeq\left(\gamma^{\prime}, a^{\prime}\right)
$$

That is, a product lottery $(\gamma, a)$ is preferred over another product lottery $\left(\gamma^{\prime}, a^{\prime}\right)$ (both having degenerate action lotteries), if the probability of getting identity-action pairs with utilities not greater than $v$ is always smaller under the first product lottery.

8 Dealing with a large set of potential members of various societies is justified by our pursuit for a general rule, to be applied to all societies. Note that Harsanyi too (like other scholars) wanted his theory to be applied to a large set of societies. The need for an infinite set of individuals is clarified in the proof of the theorem. 
Note that monotonicity is an ordinal condition that does not depend upon the choice of the numerical representation $V$.

For a given society $I$, let $\triangle(I)$ denote the set of identity lotteries over $I$.

Definition 2 Utilitarianism: The observer is a utilitarian if, for every society $I \subset \mathscr{I}$, her preferences restricted to $\Delta(I) \times \triangle(\mathscr{A})$ admit a representation of the form

$$
V(\gamma, \ell)=\sum_{i \in I} \gamma_{i} U_{i}\left(\ell_{i}\right)
$$

where $\ell_{i} \in \Delta(\mathscr{X})$ is the lottery faced by individual $i$ (i.e., $\ell_{i}(x)=$ $\left.\sum_{\{a \in \operatorname{supp}(l): a(i)=x\}} \ell(a)\right), U_{i}\left(\ell_{i}\right):=\sum_{x \in \mathscr{X}} u_{i}(x) \ell_{i}(x)$ is an expected utility (EU) representation of $\succcurlyeq_{i}$ and the functions $U_{i}$ are unique up to common positive affine transformations.

As is well known, the main behavioral property that characterizes EU preferences is independence:

Definition 3 Independence: Let $\tilde{\succcurlyeq}$ be a preference relation on $\triangle(\mathscr{X})$. Then, for all $p, q, r \in \triangle(\mathscr{X})$ and for all $\beta \in[0,1]$,

$$
p \tilde{\succcurlyeq} q \Rightarrow \beta p+(1-\beta) r \check{\succcurlyeq} \beta q+(1-\beta) r
$$

\section{Assumptions}

We make the following assumptions on $\succcurlyeq$ :

Axiom 1 Impartiality: For any two individuals $i, j \in \mathscr{I}$,

(1) for all $\ell \in \Delta(\mathscr{A}), \succcurlyeq_{i}=\succcurlyeq_{j}$ and $\ell_{i}=\ell_{j} \Rightarrow(i, \ell) \sim(j, \ell)$

(2) $\left(i, a_{\max }\right) \sim\left(j, a_{\max }\right)$ and $\left(i, a_{\min }\right) \sim\left(j, a_{\min }\right)$

Part (1) of this axiom states that, given an action lottery $\ell$, if two individuals $i$ and $j$ with identical preferences are faced with the same action lottery, then the observer is indifferent between facing $\ell$, while being individual $i$, and facing $\ell$, while being individual $j$. This requirement seems quite natural. Part (2) says that being individual $i$ and getting one of the extreme outcomes, $x_{\min }$ or $x_{\max }$, is assumed ethically equivalent to being individual $j$ and getting the (same) extreme outcome. This assumption is justified since $x_{\max }$ is of extremely high value, taken to be greater than the combined wealth of the whole society, and hence the observer has no reason to assume that at this extreme wealth she prefers imagining herself being individual $i$ over being individual $j$. Clearly, the same justification applies to the outcome $x_{\min }$ that represents a debt equal to the combined wealth of the whole society. Note that indifference need not hold for intermediate outcome values, as the observer may attain a lower utility when she imagines being a wealthier individual. A justification of a similar flavor was provided by Karni (1998) who, in a different framework, employed a stronger axiom to derive utilitarianism: "This value judgment ... is obtained by default. The methodological 
framework of revealed preference provides no ground for preferring one individual's most preferred alternative over that of the other. Consequently, strict preference in either direction is either biased or involves considerations other than the rank order of the alternatives". Segal (2000) dictatorship indifference axiom is similar to this requirement. ${ }^{9}$

Henceforth, we assume that the observer preferences satisfy the impartiality axiom. To emphasize it, we call her an impartial observer.

Axiom 2 Strong acceptance: For all $i \in \mathscr{I}$ and $\ell, \ell^{\prime} \in \Delta(\mathscr{A})$ satisfying $\forall j \neq i \quad \ell_{j}=$ $\ell_{j}^{\prime}$, if $\gamma_{i}>0$ then

$$
\ell_{i} \succcurlyeq_{i} \ell_{i}^{\prime} \Leftrightarrow(\gamma, \ell) \succcurlyeq\left(\gamma, \ell^{\prime}\right)
$$

This axiom states that the impartial observer sympathizes with individual $i$ and fully adopts his preferences when she imagines herself being this individual with a positive probability, and when all other individuals are unaffected by her choice. This axiom strengthens Harsanyi's acceptance principle, according to which this sympathy holds for $\gamma_{i}=1$. Axiom 2 also is analogous to an axiom called strong Pareto, a version of Harsanyi's Pareto principle that was used in his aggregation analysis (see Harsanyi 1955; Weymark 1991; Epstein and Segal 1992). ${ }^{10}$ To see the connection between our axiom and the strong Pareto principle note that, by sequentially applying our axiom, the following property holds: for any $\ell, \ell^{\prime} \in \Delta(\mathscr{A})$, if $\ell_{i} \succcurlyeq_{i} \ell_{i}^{\prime}$ for all $i \in \operatorname{Supp}(\gamma)$ then $(\gamma, \ell) \succcurlyeq\left(\gamma, \ell^{\prime}\right) .{ }^{11}$ In a sense, strong acceptance unifies two of Harsanyi's main ideas, taken from his two famous analyses of social choice theory. Finally, our axiom is analogous to Karni (1998) sympathy assumption.

The strong acceptance axiom enables us to express the impartial observer's function $V$ as a social welfare function. That is, $V$ can be expressed as a function $W$ that, instead of the action lottery $\ell$, depends on the individuals' utilities associated with their induced lotteries $\ell_{i}$. More formally, let $V_{i}\left(\ell_{i}\right):=V(i, \ell)$ be a representing utility the impartial observer attaches to individual $i$ preferences. Note that, by impartiality, $V_{i}\left(x_{\min }\right)=$ $V_{j}\left(x_{\min }\right):=v_{\min }$ and $V_{i}\left(x_{\max }\right)=V_{j}\left(x_{\max }\right):=v_{\max }$, for all $i, j \in \mathscr{I}$, and hence by continuity, the image of $V_{i}$, for all $i$, is equal to the closed interval [ $\left.v_{\min }, v_{\max }\right]$. Then, strong acceptance implies that $V(\gamma, \ell)$ can be written as $W(\boldsymbol{\gamma}, \mathbf{V}(\ell))$, where

9 Part (2) can be replaced by a weaker condition: there exists $i^{*}$ such that for all $j,\left(i^{*}, a_{\max }\right) \succcurlyeq\left(j, a_{\max }\right)$ and $\left(j, a_{\min }\right) \succcurlyeq\left(i^{*}, a_{\min }\right)$. Although our main result still holds under this condition, we prefer the more intuitive current version. Omitting the second part altogether would probably require strengthening some of the other axioms. This, however, is left for future research.

10 Strong Pareto: For a given society $I$, (1) for all lotteries $\ell, \ell^{\prime} \in \Delta(\mathscr{A})$, if $\ell_{i} \succcurlyeq_{i} \ell_{i}^{\prime}$ for all $i$, then $\ell \succcurlyeq \ell^{\prime}$ and (2) if, furthermore, there exists an individual $i^{\prime}$ such that $\ell_{i^{\prime}} \succ_{i^{\prime}} \ell_{i^{\prime}}^{\prime}$, then $\ell \succ \ell^{\prime}$.

11 To see it, assume without loss of generality that $\operatorname{Supp}(\gamma)=\{1,2, \ldots, n\}$ and note that

$$
\begin{aligned}
& (\gamma, \ell)=\left(\gamma,\left(\ell_{1}, \ell_{2}, \ldots, \ell_{n}\right)\right) \succcurlyeq\left(\gamma,\left(\ell_{1}^{\prime}, \ell_{2}, \ldots, \ell_{n}\right)\right) \succcurlyeq\left(\gamma,\left(\ell_{1}^{\prime}, \ell_{2}^{\prime}, \ldots, \ell_{n}\right)\right) \succcurlyeq \cdots \\
& \succcurlyeq\left(\gamma,\left(\ell_{1}^{\prime}, \ell_{2}^{\prime}, \ldots, \ell_{n}^{\prime}\right)\right)=\left(\gamma, \ell^{\prime}\right)
\end{aligned}
$$


$W$ is defined over $\triangle\left(\left[v_{\min }, v_{\max }\right]\right)$, the set of lotteries over all attainable utility values in which, for all $i \in \operatorname{Supp}(\gamma), \boldsymbol{\gamma}_{i}=\gamma_{i}$ is the probability of attaining $(\mathbf{V}(\ell))_{i}=$ $V_{i}\left(\ell_{i}\right)$. To see how $W$ is constructed assume, for expositional clarity, that $\operatorname{Supp}(\gamma)=$ $\{1, \ldots, n\}$. Then, given $V$ and $V_{i}$, for any $\gamma \in \triangle(\{1, \ldots, n\})$ and $\mathbf{v}=\left(v_{1}, \ldots, v_{n}\right) \in$ $\left[v_{\text {min }}, v_{\text {max }}\right]^{n}$, define $W$ by $W(\boldsymbol{\gamma}, \mathbf{v}):=V(\gamma, \ell)$, for the imaginary lottery $\gamma$ satisfying $\gamma_{i}=\gamma_{i}$ and for any $\ell$ satisfying $v_{i}=V_{i}\left(\ell_{i}\right)$, for all $i \in\{1, \ldots, n\}$. By strong acceptance, $W$ is well defined. Furthermore, for a given $\boldsymbol{\gamma}, W$ is monotonic increasing with respect to $v_{i}$ whenever $\boldsymbol{\gamma}_{i}>0$. Note that, by construction, $W$ satisfies $W(1, v)=$ $v$ for all $v \in\left[v_{\min }, v_{\max }\right]$ where, with slight abuse of notations, $(1, v)$ stands for getting $v$ with probability one.

The following properties will be used in the sequel.

Lemma 1 Assume the observer satisfies impartiality and strong acceptance. Then (a) for all $\ell, \ell^{\prime} \in \triangle(\mathscr{A})$,

$$
\succcurlyeq_{i}=\succcurlyeq_{j} \text { and } \ell_{i}=\ell_{j}^{\prime} \Rightarrow(i, \ell) \sim\left(j, \ell^{\prime}\right)
$$

(b) for all $(\gamma, \ell) \in \triangle(\mathscr{I}) \times \triangle(\mathscr{A})$,

$$
(i, \ell) \sim(j, \ell) \text { for all } i, j \in \operatorname{Supp}(\gamma) \Rightarrow(\gamma, \ell) \sim(k, \ell), \text { for all } k \in \operatorname{Supp}(\gamma)
$$

(c) for all $\left(\gamma^{e}, \ell\right),\left(\gamma^{e}, \ell^{\prime}\right) \in \triangle(\mathscr{I}) \times \triangle(\mathscr{A})$, where Supp $\left(\gamma^{e}\right)=\{1, \ldots, n\}$, if there exists a permutation $\pi$ on $\{1, \ldots, n\}$ such that $\left(i, \ell_{i}\right) \sim\left(\pi(i), \ell_{\pi(i)}^{\prime}\right)$ for all $i$, then

$$
\left(\gamma^{e}, \ell\right) \sim\left(\gamma^{e}, \ell^{\prime}\right)
$$

The proof appears in Sect. 6.1.

Axiom 3 Convexity: Consider an equiprobable lottery $\gamma^{e} \in \triangle(I)$ and two lotteries $\ell, \ell^{\prime} \in \triangle(\mathscr{A})$ for which there exist two individuals $i, j \in I$ satisfying $\ell_{i} \succ_{i} \ell_{i}^{\prime}$ and $\ell_{j} \prec_{j} \ell_{j}^{\prime}$. Then, for all $\beta \in(0,1)$,

$$
\left(\gamma^{e}, \ell\right) \sim\left(\gamma^{e}, \ell^{\prime}\right) \Rightarrow\left(\gamma^{e}, \beta \ell+(1-\beta) \ell^{\prime}\right) \succcurlyeq\left(\gamma^{e}, \ell\right)
$$

As was explained in Introduction, this axiom is an expression of procedural fairness and is in agreement with Diamond's critique. ${ }^{12}$

We include the requirement of having two individuals with opposing preferences since procedural fairness has greater appeal when real conflict exists. However, it is straightforward to verify that, with continuity, this requirement can be omitted. Hence, in situations where only one individual faces distinct lotteries under the action lotteries $\ell$ and $\ell^{\prime}$, convexity implies that his preferences must also be convex.

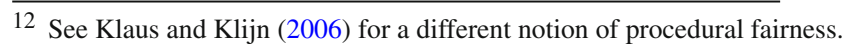


Convexity is also related to social stability. Consider a society $I \subset \mathscr{I}$, whose set of available actions is given by a finite $A \subset \mathscr{A}$. For a given identity lottery $\gamma \in \triangle(I)$, the impartial observer's aim is to find the optimal action lottery that maximizes her utility. That is, the impartial observer seeks to solve the problem

$$
\max _{\ell \in \triangle(A)} V(\gamma, \ell)
$$

For societal stability, it is desirable that the set of optimal action lotteries does not change drastically when only minor changes occur. That is, we want this set to be upper hemi-continuous and convex valued with respect to the set of available actions $A$. Clearly, the continuity of $\succcurlyeq$ implies upper hemi-continuity, while convexity is equivalent to the optimal set being a convex valued correspondence.

Axiom 4 Source indifference: For all societies $\left\{i_{1}, \ldots, i_{n}\right\}$ and for all sets of available actions $\left\{a^{1}, \ldots, a^{n}\right\}$, if there exists $k \in\{1, \ldots, n\}$ such that $\left(i_{j}, a^{k}\right) \sim\left(i_{k}, a^{j}\right)$ for all $j$, then

$$
\left(\gamma^{e}, a^{k}\right) \sim\left(i_{k}, \ell^{e}\right)
$$

where $\gamma^{e}=\sum_{j=1}^{n} \frac{1}{n} i_{j}$ and $\ell^{e}=\sum_{j=1}^{n} \frac{1}{n} a^{j}$.

To illustrate, consider the following matrix and suppose that the impartial observer

\begin{tabular}{c||cccccc} 
& $a^{1}$ & $a^{2}$ & $\cdots$ & $a^{k}$ & $\cdots$ & $a^{n}$ \\
\hline \hline$i_{1}$ & & & & $x_{1}$ & & \\
$i_{2}$ & & & & $x_{2}$ & & \\
$\vdots$ & & & & $\vdots$ & & \\
$i_{k}$ & $y^{1}$ & $y^{2}$ & $\cdots$ & $z$ & $\cdots$ & $y^{n}$ \\
$\vdots$ & & & & $\vdots$ & & \\
$i_{n}$ & & & & $x_{n}$ & &
\end{tabular}

is indifferent between the following two options, for all $j$ : (1) receiving an outcome $x_{j}$ while facing the deterministic action $a^{k}$ and imagining being individual $i_{j}$, and (2) receiving an outcome $y^{j}$ while facing the deterministic action $a^{j}$ and imagining being individual $i_{k}$. There are two ways to randomize, with equal probabilities, over these degenerate pairs of equivalent product lotteries. The product lottery $\left(\gamma^{e}, a^{k}\right)$ randomizes over identity lotteries (for the given action $a^{k}$ ), while product lottery $\left(i_{k}, \ell^{e}\right)$ randomizes over action lotteries (for the given individual $i_{k}$ ). Then, as was argued by Harsanyi in his response to Diamond and was implicitly assumed by him, the impartial observer should be indifferent between the two randomizations. We want to emphasize that, a priori, there is no clear reason to prefer either of these lotteries. Moreover, and as is explained in the next section (Comment 4), our utilitarian result 
holds even if Axiom 4 is relaxed and only requires (weak) preference of identity lotteries. $^{13}$

The following lemma shows that, given impartiality and strong acceptance, source indifference for equiprobability lotteries $\gamma^{e}$ and $\ell^{e}$ implies that this property holds for all lotteries $\gamma$ and $\ell^{\gamma}$. This property will be used later on.

Lemma 2 Assume the observer satisfies impartiality, strong acceptance and source indifference. For all societies $\left\{i_{1}, \ldots, i_{n}\right\}$ and for all sets of available actions $\left\{a^{1}, \ldots, a^{n}\right\}$, if there exists $k \in\{1, \ldots, n\}$ such that $\left(i_{j}, a^{k}\right) \sim\left(i_{k}, a^{j}\right)$ for all $j$, then, for all $\gamma=\sum_{j=1}^{n} \gamma_{i_{j}} i_{j}$ and $\ell^{\gamma}=\sum_{j=1}^{n} \gamma_{i_{j}} a^{j}$,

$$
\left(\gamma, a^{k}\right) \sim\left(i_{k}, \ell^{\gamma}\right)
$$

The proof is relegated to Sect. 6.1 .

\section{Utilitarianism}

Our main result shows that the preceding axioms force all individuals to be of the EU type and, in addition, the impartial observer must be a utilitarian. That is, the behavioral assumptions on the impartial observer preferences induce her, as well as all individuals, to satisfy the independence axiom. This is achieved without imposing independence explicitly (neither on individuals nor on the observer).

Theorem Assume the observer satisfies impartiality. Then, her preferences satisfy strong acceptance, convexity and source indifference if, and only if, all individuals in $\mathscr{I}$ satisfy independence and the observer is a utilitarian.

The proof, which is relegated to Sect. 6.2, consists of two parts. First, we prove that all individuals in $\mathscr{I}$ must satisfy the independence axiom. Then, we demonstrate that the impartial observer's preferences can be represented by a weighted average of the individual utilities.

Comment 1 Consider the Diamond example. Having the identity lottery $\gamma^{e}=\left(\frac{1}{2}, \frac{1}{2}\right)$, choosing action $a^{i}$ corresponds to the pair $\left(\gamma^{e}, a^{i}\right)$, while tossing a fair coin corresponds to the pair $\left(\gamma^{e}, \ell^{e}\right)=\left(\gamma^{e}, \frac{1}{2} a^{1}+\frac{1}{2} a^{2}\right)$. By source indifference, $\left(\gamma^{e}, a^{1}\right) \sim$ $\left(1, \ell^{e}\right)$ and $\left(\gamma^{e}, a^{1}\right) \sim\left(2, \ell^{e}\right)$. Hence, $\left(1, \ell^{e}\right) \sim\left(2, \ell^{e}\right)$ and therefore, by Lemma $1(\mathrm{~b})$, $\left(1, \ell^{e}\right) \sim\left(\gamma^{e}, \ell^{e}\right)$. But then, by transitivity, $\left(\gamma^{e}, a^{1}\right) \sim\left(\gamma^{e}, \ell^{e}\right)$ and the impartial observer is indifferent between the first action (Policy (1)) and the mixture (Policy (2)). Put differently, she does not strictly prefer tossing a fair coin over the pure action

\footnotetext{
13 It should be noted that source indifference might seem less appealing in situations in which the two randomizations are inherently less symmetric. For example, if identities are being fixed once and for a lifetime while action lotteries are repeated. Also note that preference of one type of randomization over the other can be seen as an example of what Ergin and Gul (2009) called issue or source preference. Viewing individuals as representatives of various generations, source indifference can contribute to the discussion on intergenerational ethics (see Asheim et al. 2012).
} 
$a^{1}$. Moreover, it can now be seen (proof omitted) that, by convexity, any mixture of the two actions $a^{1}$ and $a^{2}$ must be indifferent to $a^{1}$. This may seem like a significant step toward proving utilitarianism. However, the derivation of these 'straight line indifference segments' from the above extremely symmetric situation does not extend to the general case and cannot be utilized to derive a utilitarian representation.

Comment 2 As noted in Introduction, Blackorby et al. (2004) and Mongin and Pivato (2015) also derived utilitarianism without imposing independence. Although these authors work within Harsanyi's aggregation theorem framework, a comparison to our theorem seems natural and is carried out by focusing on the analysis of Mongin and Pivato (2015). Consider a given society $I$, with a set of actions $A$, and identify every product lottery $(\gamma, \ell)$ with a matrix whose rows correspond to individuals and columns correspond to actions. Mongin and Pivato's ex ante analysis is manifested by their row preference assumption, an assumption that is analogous to our strong acceptance axiom. Similarly, their ex post analysis is manifested by a column preference assumption that, in our model, would require an improvement in the impartial observer's situation whenever an action $a$ is replaced by a better action $\bar{a}$. Together with a coordinate monotonicity assumption, these two assumptions enable Mongin and Pivato to employ Gorman (1968) separability theorem and derive a fully separable representation of the observer preferences. As can be seen in Sect. 6.2, our proof uses different arguments. Nevertheless, one might conjecture that, since source indifference implies similar treatment of columns and rows then, together with strong acceptance, Gorman's separability theorem could be applied to yield our result. However, this is not true. As can be seen in Examples 1 and 2 below, strong acceptance and source indifference are not sufficient to imply utilitarianism.

Comment 3 Another result that is close to ours appears in GKPS (2010). Their Theorem 3 roughly states that an observer is a utilitarian if and only if she satisfies acceptance, independence over identity lotteries and (their notion) of source indifference. To distinguish our result from theirs, note that we derive utilitarianism without assuming any form of independence. ${ }^{14}$

The following first two examples demonstrate the necessity of convexity. The third demonstrates the necessity of source indifference.

Example 1 Here we present a non-utilitarian impartial observer who satisfies all axioms except for convexity. Assume that all preferences $\succcurlyeq_{i}$ of individuals $i \in \mathscr{I}$ belong to the rank-dependent utility class (RDU; see Weymark 1981; Quiggin 1982). Let $g:[0,1] \rightarrow[0,1]$ be an increasing and onto function. For a given simple lottery $r$ and $z \in \operatorname{Supp}(r)$ define $F_{r}(z):=\sum_{y \leqslant z} r(y), F_{r}\left(z_{-}\right):=\sum_{y<z} r(y)$ and $\nabla g(z ; r):=g\left(F_{r}(z)\right)-g\left(F_{r}\left(z_{-}\right)\right)$. On simple lotteries, RDU preferences are represented by a function of the form $V(p)=\sum_{x} u(x) \nabla g(x ; p)$. When $g$ is the identity function, $\nabla g(x ; p)=p(x)$ and RDU preferences are reduced to EU preferences. We assume that, in the eyes of the impartial observer, individual $i$ 's preferences are represented by $V_{i}(p)=\sum_{x} u_{i}(x) \nabla g(x ; p)$, where $g$ is common to all individuals and,

14 It should also be noted that the notion of source indifference used by GKPS (2010) (they termed it 'indifference between identity and action lotteries') is stronger than ours. This is formally stated as Lemma 4 (see Sect. 6.4). 
for all $i, j \in \mathscr{I}, u_{i}\left(x_{\min }\right)=u_{j}\left(x_{\min }\right)$ and $u_{i}\left(x_{\max }\right)=u_{j}\left(x_{\max }\right)$. The observer preferences are also of the RDU type and are represented by

$$
V^{r}(\gamma, \ell)=\sum_{i \in I} V_{i}\left(\ell_{i}\right) \nabla g\left(V_{i}\left(\ell_{i}\right) ; \gamma\right)
$$

Impartiality and strong acceptance are satisfied by construction. To verify that source indifference is satisfied consider, without loss of generality, a society $I=$ $\{1, \ldots, n\}$, a set of available actions $\left\{a^{1}, \ldots, a^{n}\right\}$ and assume that there exists $k$ for which $V^{r}\left(j, a^{k}\right)=V^{r}\left(k, a^{j}\right)$ for all $j$. Then, for all $j$,

$$
u_{j}\left(a^{k}(j)\right)=V_{j}\left(a^{k}(j)\right)=V^{r}\left(j, a^{k}\right)=V^{r}\left(k, a^{j}\right)=V_{k}\left(a^{j}(k)\right)=u_{k}\left(a^{j}(k)\right)
$$

Hence,

$$
\begin{aligned}
V^{r}\left(\gamma^{e}, a^{k}\right) & =\sum_{j \in I} u_{j}\left(a^{k}(j)\right) \nabla g\left(u_{j}\left(a^{k}(j)\right) ; \gamma^{e}\right) \\
& =\sum_{j \in I} u_{k}\left(a^{j}(k)\right) \nabla g\left(u_{j}\left(a^{k}(j)\right) ; \gamma^{e}\right) \\
& =\sum_{j \in I} u_{k}\left(a^{j}(k)\right) \nabla g\left(a^{j}(k) ; \ell_{k}^{e}\right)=V^{r}\left(k, \ell^{e}\right)
\end{aligned}
$$

as required.

To see that convexity does not hold assume that $g$ is strictly concave and fix $j \in I$. Let $\ell, \ell^{\prime} \in \Delta(\mathscr{A})$ be two distinct action lotteries satisfying $\ell_{i}=\ell_{i}^{\prime}$ for all $i \neq j$, $\ell_{j} \neq \ell_{j}^{\prime}$ and $V_{j}\left(\ell_{j}\right)=V_{j}\left(\ell_{j}^{\prime}\right)$ (clearly, such lotteries exist). The strict concavity of $g$ implies $V_{j}\left(\frac{1}{2} \ell_{j}+\frac{1}{2} \ell_{j}^{\prime}\right)<V_{j}\left(\ell_{j}\right)$ and hence, for any $\gamma$ with $\gamma_{j}>0$, $V^{r}\left(\gamma, \frac{1}{2} \ell+\frac{1}{2} \ell^{\prime}\right)<V^{r}(\gamma, \ell) .{ }^{15}$

15 Perhaps the simplest way to see it is to observe that, for continuous lotteries, $V_{j}\left(\ell_{j}\right)=$ $\int_{z} u_{j}(z) \mathrm{d} g\left(F_{\ell_{j}}(z)\right)=u_{j}\left(x_{\max }\right)-\int_{z} g\left(F_{\ell_{j}}(z)\right) u_{j}^{\prime}(z) \mathrm{d} z$. Therefore

$$
\begin{aligned}
V_{j}\left(\frac{1}{2} \ell_{j}+\frac{1}{2} \ell_{j}^{\prime}\right)= & u_{j}\left(x_{\max }\right)-\int_{z} g\left(F_{\frac{1}{2} \ell_{j}+\frac{1}{2} \ell_{j}^{\prime}}(z)\right) u_{j}^{\prime}(z) \mathrm{d} z \\
= & u_{j}\left(x_{\max }\right)-\int_{z} g\left(\frac{1}{2} F_{\ell_{j}}(z)+\frac{1}{2} F_{\ell_{j}^{\prime}}(z)\right) u_{j}^{\prime}(z) \mathrm{d} z \\
< & u_{j}\left(x_{\max }\right)-\int_{z}\left[\frac{1}{2} g\left(F_{\ell_{j}}(z)\right)+\frac{1}{2} g\left(F_{\ell_{j}^{\prime}}(z)\right)\right] u_{j}^{\prime}(z) \mathrm{d} z \\
= & \frac{1}{2}\left[u_{j}\left(x_{\max }\right)-\int_{z} g\left(F_{\ell_{j}}(z)\right) u_{j}^{\prime}(z) \mathrm{d} z\right] \\
& +\frac{1}{2}\left[u_{j}\left(x_{\max }\right)-\int_{z} g\left(F_{\ell_{j}^{\prime}}(z)\right) u_{j}^{\prime}(z) \mathrm{d} z\right] \\
= & \frac{1}{2} V_{j}\left(\ell_{j}\right)+\frac{1}{2} V_{j}\left(\ell_{j}^{\prime}\right)=V_{j}\left(\ell_{j}\right)
\end{aligned}
$$


Note that, as the following case shows, non-convexity of $\succcurlyeq_{i}$ (which is manifested by the concavity of $g$ ) is not necessary for the non-convexity of $\succcurlyeq$. For this, let $I=$ $\{1, \ldots, 5\}$ and consider the two actions described by the matrix (the entries are the utility values)

\begin{tabular}{c||cc} 
& $a^{1}$ & $a^{2}$ \\
\hline \hline 1 & 1 & 0 \\
2 & 0 & 1 \\
3 & 1 & 1 \\
4 & 1 & 1 \\
5 & 1 & 1
\end{tabular}

Let $g$ be given by the convex piecewise linear function

$$
g(t)= \begin{cases}0 & t \leqslant 0.2 \\ -\frac{1}{4}+\frac{5}{4} t & \text { otherwise }\end{cases}
$$

and note that, by the convexity of $g$, each $\succcurlyeq_{i}$ is convex.

Clearly, for both $j=1,2$,

$$
V^{r}\left(\gamma^{e}, a^{j}\right)=g(0.2) \times 0+(1-g(0.2)) \times 1=1
$$

Next, consider the lottery $\frac{1}{2} a^{1}+\frac{1}{2} a^{2}$. For $i \in\{1,2\}$,

$$
V_{i}\left(\frac{1}{2} a^{1}(i)+\frac{1}{2} a^{2}(i)\right)=g(0.5) \times 0+(1-g(0.5)) \times 1=\frac{5}{8}
$$

while, for $i \in\{3,4,5\}, V_{i}\left(\frac{1}{2} a^{1}(i)+\frac{1}{2} a^{2}(i)\right)=1$. Hence, for the impartial observer,

$$
\begin{aligned}
V^{r}\left(\gamma^{e}, \frac{1}{2} a^{1}+\frac{1}{2} a^{2}\right) & =g(0.4) \times \frac{5}{8}+(1-g(0.4)) \times 1 \\
& =\frac{1}{4} \times \frac{5}{8}+\frac{3}{4} \times 1=\frac{29}{32}<1
\end{aligned}
$$

and convexity is not satisfied.

Example 2 In the two cases described in Example 1, either individual preferences are non-convex with respect to outcome lotteries (when $g$ is concave) or the impartial observer preferences are non-convex with respect to identity lotteries (when $g$ is convex). This might suggest that convexity would be satisfied if all preferences involved were convex. As we now show, this conjecture is false. 
Assume that individual preferences are weighted utility (WU; see Chew 1983). That is, for all $i$ and $p \in \triangle(\mathscr{X})$,

$$
V_{i}(p)=V(p)=\sum_{k} p_{k} \frac{w\left(x_{k}\right)}{\sum_{j} p_{j} w\left(x_{j}\right)} u\left(x_{k}\right)
$$

where $u$ is a strictly increasing utility function and $w$ is a non-constant and positive weighting function. These preferences belong to the betweenness class (see Chew 1989; Dekel 1986), a class that is characterized by the property: for all lotteries $p$ and $q, p \succcurlyeq q$ if and only if $p \succcurlyeq \lambda p+(1-\lambda) q \succcurlyeq q$, for all $\lambda \in(0,1)$. Clearly, betweenness implies that WU preferences are convex.

The impartial observer preferences are of the same type and are given by

$$
V^{w}(\gamma, \ell)=\sum_{i} \gamma_{i} \frac{w\left(u^{-1}\left(V\left(\ell_{i}\right)\right)\right)}{\sum_{j} \gamma_{j} w\left(u^{-1}\left(V\left(\ell_{j}\right)\right)\right)} V\left(\ell_{i}\right)
$$

As in Example 1, source indifference is satisfied. To see it, assume (for $k=1$ ) $V^{w}\left(j, a^{1}(j)\right)=V^{w}\left(1, a^{j}(1)\right)$, for all $j$. That is, $u\left(a^{1}(j)\right)=u\left(a^{j}(1)\right)$ or, equivalently, $a^{1}(j)=a^{j}(1)$, for all $j$. Then

$$
\begin{aligned}
V^{w}\left(\gamma^{e}, a^{1}\right) & =\sum_{i} \frac{1}{n} \frac{w\left(\left(u^{-1} \circ u\right)\left(a^{1}(i)\right)\right)}{\sum_{j} \frac{1}{n} w\left(\left(u^{-1} \circ u\right)\left(a^{1}(j)\right)\right)} u\left(a^{1}(i)\right) \\
& =\sum_{i} \frac{1}{n} \frac{w\left(a^{1}(i)\right)}{\sum_{j} \frac{1}{n} w\left(a^{1}(j)\right)} u\left(a^{1}(i)\right) \\
& =\sum_{i} \frac{1}{n} \frac{w\left(a^{i}(1)\right)}{\sum_{j} \frac{1}{n} w\left(a^{j}(1)\right)} u\left(a^{i}(1)\right) \\
& =V^{w}\left(1, \ell^{e}\right)
\end{aligned}
$$

Next we show that convexity is not satisfied. Consider again the Diamond example. Assume that $u(x)=x$ and $w(x)=2+x .^{16}$ Then,

$$
V^{w}\left(\gamma^{e}, a^{1}\right)=\frac{\frac{1}{2} w(1)}{\frac{1}{2} w(1)+\frac{1}{2} w(0)}=\frac{0.5 \times 3}{0.5 \times 3+0.5 \times 2}=\frac{3}{5}
$$

and

$$
V^{w}\left(\gamma^{e}, a^{2}\right)=\frac{\frac{1}{2} w(1)}{\frac{1}{2} w(0)+\frac{1}{2} w(1)}=\frac{0.5 \times 3}{0.5 \times 2+0.5 \times 3}=\frac{3}{5}
$$

\footnotetext{
16 WU preferences increase with respect to first-order stochastic-dominance when $w$ and $w \cdot u$ are bounded on the outcome interval $([0,1]$ in this example) and when $w(x)(u(x)-u(s))$ is monotonic increasing in $x$ for all $s \in[0,1]$. Clearly, these conditions are satisfied.
} 
Let $\ell=0.8 a^{1}+0.2 a^{2}$ be a mixture of $a^{1}$ and $a^{2}$. Then,

$$
\begin{gathered}
V\left(\ell_{1}\right)=\frac{0.8 w(1)}{0.8 w(1)+0.2 w(0)}=\frac{0.8 \times 3}{0.8 \times 3+0.2 \times 2}=\frac{6}{7} \\
V\left(\ell_{2}\right)=\frac{0.2 w(1)}{0.8 w(0)+0.2 w(1)}=\frac{0.2 \times 3}{0.8 \times 2+0.2 \times 3}=\frac{3}{11}
\end{gathered}
$$

and, for the impartial observer,

$$
\begin{aligned}
V^{w}\left(\gamma^{e}, \ell\right)= & \frac{1}{2} \frac{w\left(u^{-1}\left(V\left(\ell_{1}\right)\right)\right)}{\frac{1}{2} w\left(u^{-1}\left(V\left(\ell_{1}\right)\right)\right)+\frac{1}{2} w\left(u^{-1}\left(V\left(\ell_{2}\right)\right)\right)} V\left(\ell_{1}\right) \\
& +\frac{1}{2} \frac{w\left(u^{-1}\left(V\left(\ell_{2}\right)\right)\right)}{\frac{1}{2} w\left(u^{-1}\left(V\left(\ell_{2}\right)\right)\right)+\frac{1}{2} w\left(u^{-1}\left(V\left(\ell_{2}\right)\right)\right)} V\left(\ell_{2}\right) \\
= & \frac{\frac{20}{7}}{\frac{20}{7}+\frac{25}{11}} \times \frac{6}{7}+\frac{\frac{25}{11}}{\frac{20}{7}+\frac{25}{11}} \times \frac{3}{11} \\
= & \frac{44}{79} \times \frac{6}{7}+\frac{35}{79} \times \frac{3}{11} \approx 0.598<\frac{3}{5}
\end{aligned}
$$

Hence, convexity is violated.

Example 3 A non-utilitarian impartial observer who satisfies all axioms except for source indifference is the generalized utilitarian impartial observer of GKPS (2010). Consider

$$
V^{g}(\gamma, \ell)=\sum_{i \in I} \gamma_{i} \phi_{i}\left[U_{i}\left(\ell_{i}\right)\right]
$$

where $\phi_{i}:\left[v_{\min }, v_{\max }\right] \rightarrow \mathbb{R}$ are strictly concave, for all $i$. It is easy to verify that strong acceptance and convexity are satisfied while, as was shown in GKPS, this observer deems identity lotteries inferior to action lotteries.

Comment 4 Consider the following assumption, which is weaker than source indifference.

Preference for identity lotteries: For all societies $\left\{i_{1}, \ldots, i_{n}\right\}$ and for all sets of available actions $\left\{a^{1}, \ldots, a^{n}\right\}$, if there exists $k \in\{1, \ldots, n\}$ such that $\left(i_{j}, a^{k}\right) \sim\left(i_{k}, a^{j}\right)$ for all $j$, then

$$
\left(\gamma^{e}, a^{k}\right) \succcurlyeq\left(i_{k}, \ell^{e}\right)
$$

In Sect. 6.3 (Lemma 3) we show that this assumption, in conjunction with strong acceptance and convexity, implies source indifference. Therefore, our theorem could be stated in a slightly stronger form. The current form is chosen because, having no reason to prefer either type of these lotteries, source indifference seems the more natural choice. Moreover, it is more in line with Harsanyi's own arguments. 


\section{Conclusion}

As stated in Introduction we argue that when societal decisions are at stake, postulates must be drawn from society-centered behavior. We have chosen to focus on the notion of procedural fairness (exhibited by convexity) and added to it the requirement that the impartial observer is indifferent between identity and action lotteries. In our main result we have shown that these two assumptions (together with strong acceptance) were sufficient to force the impartial observer to be a utilitarian. Unlike most utilitarian results, no form of the independence axiom was required here.

In addition to offering a society-centered basis for utilitarianism, our result sheds more light on what is needed in order to always have a strict preference for procedural fairness. Since preference for identity lotteries implies source indifference (Lemma 3, Sect. 6.3), then, in order to have a strict preference for procedural fairness, the impartial observer must display a preference for action lotteries. Two such non-utilitarian models exist in the literature. The first follows from Karni and Safra (2000). ${ }^{17}$ In their model, which leads to the representation $V(\gamma, \ell)=\sum_{i \in I} \gamma_{i} V_{i}\left(\ell_{i}\right)$, individuals possess a sense of justice and preference for procedural fairness is solely manifested by their behavior (their utilities $V_{i}$ are assumed to be concave). It can easily be verified that this impartial observer displays a preference for action lotteries. The second model is the generalized utilitarian impartial observer of GKPS (2010). As mentioned above, GKPS show that a preference for action lotteries holds if and only if each $\phi_{i}$ is concave, a condition that implies procedural fairness. For a third model, consider a rank dependent, or a Gini, impartial observer, whose preferences are represented by

$$
V^{r d}(\gamma, \ell)=\sum_{i \in I} \phi\left(U_{i}\left(\ell_{i}\right)\right) \nabla g\left(U_{i}\left(\ell_{i}\right) ; \gamma\right)
$$

(where each $U_{i}$ is of the EU type and both $\phi$ and $g$ are concave). As can easily be verified, a preference for action lotteries follows from Chew et al. (1987) while procedural fairness follows from Quiggin (1993, Section 9.1).

\section{Proofs}

\subsection{Proofs of Lemmas 1 and 2}

Proof of Lemma 1 (a) Assume $\succcurlyeq_{i}=\succcurlyeq_{j}$ and consider $\ell, \ell^{\prime} \in \triangle(\mathscr{A})$ satisfying $\ell_{i}=\ell_{j}^{\prime}$. Construct an action lottery $\bar{\ell}$ that satisfies $\bar{\ell}_{i}=\bar{\ell}_{j}=\ell_{i}=\ell_{j}^{\prime}$. Then

$$
(i, \ell) \sim(i, \bar{\ell}) \sim(j, \bar{\ell}) \sim\left(j, \ell^{\prime}\right)
$$

as required (the first and the last indifferences follow from strong acceptance while the second follows from impartiality).

17 See also Grant et al. (2012). 
(b) Let $v=V(i, \ell)=V_{i}\left(\ell_{i}\right)$ and note that, by the arguments that precede the statement of the lemma, $V(\gamma, \ell)=W(\gamma,(v, \ldots, v))$ while $V(k, \ell)=W(1, v)$. That is, the product lottery $(\gamma, \ell)$ is equivalent to a utility lottery with $n$ identical outcomes (where $n$ is the number of elements in $\operatorname{Supp}(\gamma)$ ), all equal to $v$, while $(k, \ell)$ is equivalent to the degenerate lottery that yields $v$ for sure. The two utility lotteries seem identical but, in order to show that the impartial observer is indeed indifferent between them, the monotonicity property must be employed.

For this, let $c_{i}\left(\ell_{i}\right) \in \mathscr{X}$ be individual $i$ 's certainty equivalent of the lottery $\ell_{i}$ (that is, $\left.c_{i}\left(\ell_{i}\right) \sim_{i} \ell_{i}\right)$ and consider the action $\hat{a}$ satisfying $\hat{a}(i)=c_{i}\left(\ell_{i}\right)$. By strong acceptance, $(\gamma, \ell) \sim(\gamma, \hat{a})$ and $(k, \ell) \sim(k, \hat{a})$. Then, as the unique utility value attained by both $(\gamma, \hat{a})$ and $(k, \hat{a})$ is $v$, monotonicity implies that $(\gamma, \hat{a}) \sim(k, \hat{a})$. By transitivity, $(\gamma, \ell) \sim(k, \ell)$.

(c) Let $\left(\gamma^{e}, \ell\right),\left(\gamma^{e}, \ell^{\prime}\right)$ and $\pi$ satisfy the conditions of the lemma. Construct two actions $\hat{a}$ and $\hat{a}^{\prime}$ satisfying $\hat{a}(i)=c_{i}\left(\ell_{i}\right)$ and $\hat{a}^{\prime}(i)=c_{i}\left(\ell_{i}^{\prime}\right)$ where, as above, $c_{i}$ is the certainty equivalent function of individual $i$. By strong acceptance, $\left(\gamma^{e}, \ell\right) \sim\left(\gamma^{e}, \hat{a}\right)$ and $\left(\gamma^{e}, \ell^{\prime}\right) \sim\left(\gamma^{e}, \hat{a}^{\prime}\right)$. The conditions $\left(i, \ell_{i}\right) \sim\left(\pi(i), \ell_{\pi(i)}^{\prime}\right)$ imply $V\left(i, \ell_{i}\right)=$ $V\left(\pi(i), \ell_{\pi(i)}^{\prime}\right)$ for all $i$, and hence,

$$
\begin{aligned}
V(i, \hat{a}) & =V\left(i, c_{i}\left(\ell_{i}\right)\right)=V\left(i, \ell_{i}\right)=V\left(\pi(i), \ell_{\pi(i)}^{\prime}\right) \\
& =V\left(\pi(i), c_{\pi(i)}\left(\ell_{\pi(i)}^{\prime}\right)\right)=V\left(\pi(i), \hat{a}^{\prime}\right)
\end{aligned}
$$

By monotonicity, $\left(\gamma^{e}, \hat{a}\right) \sim\left(\gamma^{e}, \hat{a}^{\prime}\right)$ and, by transitivity, $\left(\gamma^{e}, \ell\right) \sim\left(\gamma^{e}, \ell^{\prime}\right)$.

Proof of Lemma 2 Consider, without loss of generality, a society $I=\{1, \ldots, n\}$, a set of available actions $A=\left\{a^{1}, \ldots, a^{n}\right\}$ and assume that (again, without loss of generality) $\left(i, a^{1}\right) \sim\left(1, a^{i}\right)$, for all $i$. Let $\gamma=\left(\gamma_{1}, \ldots, \gamma_{n}\right)$.

First assume that $\gamma$ is rational. That is, $\gamma_{i}=\frac{n_{i}}{m_{i}}$, for all $i$. Consider a new society $\bar{I}=\{\overline{1}, \overline{2}, \ldots\}$ with $m_{1} \cdots m_{n}$ individuals, in which the first $n_{1} m_{2} \cdots m_{n}$ individuals are identical to individual 1 of $I$, the next $m_{1} n_{2} m_{3} \cdots m_{n}$ individuals are identical to individual 2 of $I$, and so on. Similarly, let the set of actions $\bar{A}=\left\{\bar{a}^{1}, \bar{a}^{2}, \ldots\right\}$ consists of $m_{1} \cdots m_{n}$ actions, in which the first $n_{1} m_{2} \cdots m_{n}$ actions are identical to action $a^{1}$ of $A$, the next $m_{1} n_{2} m_{3} \cdots m_{n}$ actions are identical to action $a^{2}$ of $A$, and so on. Finally, let $\bar{\gamma}^{e}$ and $\bar{\ell}^{e}$ be the equiprobability lotteries over $\bar{I}$ and $\bar{A}$, respectively. By construction, $\left(\bar{l}, \bar{a}^{1}\right) \sim\left(\overline{1}, \bar{a}^{\bar{l}}\right)$, for all $\bar{l}$. By source indifference, $\left(\bar{\gamma}^{e}, \bar{a}^{1}\right) \sim\left(\overline{1}, \bar{\ell}^{e}\right)$. To conclude note that, by monotonicity, $\left(\gamma, a^{1}\right) \sim\left(\bar{\gamma}^{e}, \bar{a}^{1}\right)$ and, by Lemma 1(a), $\left(\overline{1}, \bar{\ell}^{e}\right) \sim\left(1, \ell^{\gamma}\right)$. Transitivity then implies $\left(\gamma, a^{1}\right) \sim\left(1, \ell^{\gamma}\right)$.

Next consider any $\gamma$ and let $\beta_{k} \rightarrow_{k \rightarrow \infty} \gamma$ be a sequence of rational lotteries that converge to $\gamma$. By construction, $\left(\beta_{k}, a^{1}\right) \rightarrow_{k \rightarrow \infty}\left(\gamma, a^{1}\right)$ and $\left(1, \ell^{\beta_{k}}\right) \rightarrow_{k \rightarrow \infty}$ $\left(1, \ell^{\gamma}\right)$. By the argument above, $\left(\beta_{k}, a^{1}\right) \sim\left(1, \ell^{\beta_{k}}\right)$ for all $k$ and hence, by continuity, $\left(\gamma, a^{1}\right) \sim\left(1, \ell^{\gamma}\right)$. 


\subsection{Proof of the Theorem}

The 'if' part is immediate. The proof of the converse is divided into two parts.

Part $I^{18}$ In this part we show that all individuals satisfy the independence axiom. Consider an individual $i^{*} \in \mathscr{I}$ and denote his preferences by $\succcurlyeq *$. We want to demonstrate that for all $p, q, r \in \triangle(\mathscr{X}), p \sim^{*} q \Rightarrow \frac{1}{2} p+\frac{1}{2} r \sim^{*} \frac{1}{2} q+\frac{1}{2} r$. This, using Herstein and Milnor (1953), would imply that $\succcurlyeq^{*}$ satisfies the independence axiom. Using the continuity of $\succcurlyeq^{*}$, we can restrict attention to equiprobability lotteries with the same number of outcomes: $p=\left(\left(\frac{1}{k}, \ldots, \frac{1}{k}\right),\left(x_{1}, \ldots, x_{k}\right)\right), q=\left(\left(\frac{1}{k}, \ldots, \frac{1}{k}\right),\left(y_{1}, \ldots, y_{k}\right)\right)$, and $r=\left(\left(\frac{1}{k}, \ldots, \frac{1}{k}\right),\left(z_{1}, \ldots, z_{k}\right)\right)$ (to see it, note that (1) any lottery with rational probabilities can be replicated by an equiprobability lottery with not necessarily distinct outcomes and (2) the set of lotteries with rational probabilities is dense in the space of all lotteries).

Consider a society $I$ consisting of $n=2 k$ individuals, all with preferences $\succcurlyeq_{i}$ $=\succcurlyeq^{*}$. Let $\pi_{1}=(1,2, \ldots, n), \pi_{2}=(2,3, \ldots, 1), \ldots, \pi_{n}=(n, 1,2, \ldots, n-1)$ be permutations on $\{1, \ldots, n\}$ (where $\pi_{j}(i)$ stands for the $i$ th element of the permutation $\pi_{j}$ ). We concentrate on a set of actions $\dot{A}=\left\{\dot{a}^{1}, \ldots, \dot{a}^{n}\right\}$ available to the society that are defined as follows: for $j=1, \ldots, k$

$$
\dot{a}^{j}(i)= \begin{cases}x_{\pi_{j}(i)} & \text { if } 1 \leqslant i \leqslant k \\ z_{\pi_{j}(i-k)} & \text { if } k<i \leqslant n\end{cases}
$$

and, for $j=k+1, \ldots, n$

$$
\dot{a}^{j}(i)= \begin{cases}z_{\pi_{j-k}(i)} & \text { if } 1 \leqslant i \leqslant k \\ x_{\pi_{j-k}(i-k)} & \text { if } k<i \leqslant n\end{cases}
$$

To illustrate, look at the following matrix

\begin{tabular}{c||cccccccc} 
& $\dot{a}^{1}$ & $\dot{a}^{2}$ & $\cdots$ & $\dot{a}^{k}$ & $\dot{a}^{k+1}$ & $\dot{a}^{k+2}$ & $\cdots$ & $\dot{a}^{n}$ \\
\hline \hline 1 & $x_{1}$ & $x_{2}$ & $\cdots$ & $x_{k}$ & $z_{1}$ & $z_{2}$ & $\cdots$ & $z_{k}$ \\
2 & $x_{2}$ & $x_{3}$ & $\cdots$ & $x_{1}$ & $z_{2}$ & $z_{3}$ & $\cdots$ & $z_{1}$ \\
$\vdots$ & $\vdots$ & $\vdots$ & $\ddots$ & $\vdots$ & $\vdots$ & $\vdots$ & $\ddots$ & $\vdots$ \\
$k$ & $x_{k}$ & $x_{1}$ & $\cdots$ & $x_{k-1}$ & $z_{k}$ & $z_{1}$ & $\cdots$ & $z_{k-1}$ \\
$k+1$ & $z_{1}$ & $z_{2}$ & $\cdots$ & $z_{k}$ & $x_{1}$ & $x_{2}$ & $\cdots$ & $x_{k}$ \\
$k+2$ & $z_{2}$ & $z_{3}$ & $\cdots$ & $z_{1}$ & $x_{2}$ & $x_{3}$ & $\cdots$ & $x_{1}$ \\
$\vdots$ & $\vdots$ & $\vdots$ & $\ddots$ & $\vdots$ & $\vdots$ & $\vdots$ & $\ddots$ & $\vdots$ \\
$n$ & $z_{k}$ & $z_{1}$ & $\cdots$ & $z_{k-1}$ & $x_{k}$ & $x_{1}$ & $\cdots$ & $x_{k-1}$
\end{tabular}

Fact $1\left(\gamma^{e}, \ell^{e}\right) \sim\left(\gamma^{e}, \dot{a}^{1}\right)$ where $\gamma^{e}=\sum_{i=1}^{n} \frac{1}{n} i$ and $\ell^{e}=\sum_{j=1}^{n} \frac{1}{n} \dot{a}^{j}$

\footnotetext{
18 The proof of this part is similar to that of Dekel et al. (1991, Theorem 2). However, dealing with social multi-person framework, our proof is more general than (and improves upon) theirs.
} 
Since for all $i, j \ell_{i}^{e}=\ell_{j}^{e}$, impartiality implies $\left(i, \ell^{e}\right) \sim\left(j, \ell^{e}\right)$ and hence, by Lemma $1(\mathrm{~b}),\left(\gamma^{e}, \ell^{e}\right) \sim\left(1, \ell^{e}\right)$. Next, since $\dot{a}^{j}(1)=\dot{a}^{1}(j)\left(x_{j}\right.$ if $j \leqslant k$ and $z_{j-k}$ otherwise $)$ then, in both $\left(1, \dot{a}^{j}\right)$ and $\left(j, \dot{a}^{1}\right)$, the impartial observer faces the same deterministic outcome. By Lemma 1 (a), $\left(1, \dot{a}^{j}\right) \sim\left(j, \dot{a}^{1}\right)$ for all $j \in I$ and, by source indifference, $\left(1, \ell^{e}\right) \sim\left(\gamma^{e}, \dot{a}^{1}\right)$. Transitivity then implies $\left(\gamma^{e}, \ell^{e}\right) \sim\left(\gamma^{e}, \dot{a}^{1}\right)$.

Fact 2 Let $\ell^{k}=\frac{1}{k} \sum_{j=1}^{k} \dot{a}^{j}$. Then $\left(\gamma^{e}, \ell^{k}\right) \sim\left(\gamma^{e}, \ell^{e}\right)$.

Since all actions $\dot{a}^{i}$ yield the same outcomes then, using impartiality and monotonicity, $\left(\gamma^{e}, \dot{a}^{i}\right) \sim\left(\gamma^{e}, \dot{a}^{1}\right)$ for all $i$. By repeated application of convexity, $\left(\gamma^{e}, \ell^{k}\right)=$ $\left(\gamma^{e}, \frac{1}{k} \sum_{j=1}^{k} \dot{a}^{j}\right) \succcurlyeq\left(\gamma^{e}, \dot{a}^{1}\right) .{ }^{19}$ Hence, by Fact 1 and transitivity, $\left(\gamma^{e}, \ell^{k}\right) \succcurlyeq\left(\gamma^{e}, \ell^{e}\right)$.

For the converse, consider the action lottery $\hat{\ell}^{k}=\frac{1}{k} \sum_{j=k+1}^{n} \dot{a}^{j}$. For all $i=$ $1, \ldots, k, \hat{\ell}_{i}^{k}$, the lottery individual $i$ faces under $\hat{\ell}^{k}$, is identical to $\ell_{k+i}^{k}$, the lottery that individual $k+i$ faces under $\ell^{k}$. By Lemma 1 (a), $\left(i, \hat{\ell}_{i}^{k}\right) \sim\left(k+i, \ell_{k+i}^{k}\right)$. Similarly, $\hat{\ell}_{k+i}^{k}$, the lottery individual $k+i$ faces under $\hat{\ell}^{k}$, is identical to $\ell_{i}^{k}$, the lottery that individual $i$ faces under $\ell^{k}$ and hence, by Lemma $1(\mathrm{a}),\left(k+i, \hat{\ell}_{k+i}^{k}\right) \sim\left(i, \ell_{i}^{k}\right)$. Therefore, by Lemma $1(\mathrm{c}),\left(\gamma^{e}, \hat{\ell}^{k}\right) \sim\left(\gamma^{e}, \ell^{k}\right)$. Since $\ell^{e}=\frac{1}{2} \hat{\ell}^{k}+\frac{1}{2} \ell^{k}$, convexity implies $\left(\gamma^{e}, \ell^{e}\right) \succcurlyeq\left(\gamma^{e}, \ell^{k}\right)$.

Hence, $\left(\gamma^{e}, \ell^{k}\right) \sim\left(\gamma^{e}, \ell^{e}\right)$.

Fact $3 \frac{1}{2} p+\frac{1}{2} r \sim * \frac{1}{2} q+\frac{1}{2} r$.

By the first part of the proof of Fact $1,\left(\gamma^{e}, \ell^{e}\right) \sim\left(1, \ell^{e}\right)$. Therefore, using transitivity and Fact $2,\left(\gamma^{e}, \ell^{k}\right) \sim\left(1, \ell^{e}\right)$. Note that in the first lottery, the first $k$ individuals face the lottery $p$ and the rest face the lottery $r$ while, in the second, individual 1 is faced with the lottery $\frac{1}{2} p+\frac{1}{2} r$.

Next consider the same set of individuals $I$ with another set of actions $\tilde{A}=$ $\left\{\tilde{a}^{1}, \ldots, \tilde{a}^{2 k}\right\}$, that is derived from $\dot{A}$ by replacing every $x_{j}$ by $y_{j}$. Clearly, a similar conclusion holds: the impartial observer is indifferent between the product lottery $\left(\gamma^{e}, \tilde{\ell}^{k}\right)$, in which the first $k$ individuals face the lottery $q$ and the rest face the lottery $r$, and the product lottery $\left(1, \tilde{\ell}^{e}\right)$, in which individual 1 is faced with the lottery $\frac{1}{2} q+\frac{1}{2} r$. But as $p \sim^{*} q$, all individuals in $I$ are indifferent between $p$ and $q$ and hence, by strong acceptance, $\left(\gamma^{e}, \ell^{k}\right) \sim\left(\gamma^{e}, \tilde{\ell}^{k}\right)$. By transitivity, $\left(1, \ell^{e}\right) \sim\left(1, \tilde{\ell}^{e}\right)$. Hence, the impartial observer, while imagining herself being individual 1 , is indifferent between the lotteries $\frac{1}{2} p+\frac{1}{2} r$ and $\frac{1}{2} q+\frac{1}{2} r$. By strong acceptance, $\frac{1}{2} p+\frac{1}{2} r \sim^{*} \frac{1}{2} q+\frac{1}{2} r$.

To conclude Part I, note that allowing $k$ to go to infinity implies that $\succcurlyeq *$ satisfies independence over the entire set of lotteries $\triangle(\mathscr{X}) .^{20}$

19 Note that by continuity, the convexity axiom holds even when there are no opposing individuals (see Sect. 3, right after the statement of the convexity axiom).

20 This is where we make use of the infinity of the set $\mathscr{I}$. 
Part II In the second part we show that the impartial observer is a utilitarian. Consider a society $I$ (without loss of generality, $I=\{1, \ldots, n\}$ ) and let $V(\gamma, \ell)$ be a representation of the impartial observer preferences where $V(i, \ell)=V_{i}\left(\ell_{i}\right)=\varphi_{i}\left(U_{i}\left(\ell_{i}\right)\right)$, $\varphi_{i}$ is monotonic increasing and, by Part I, $U_{i}\left(\ell_{i}\right)=\sum_{x \in \mathscr{X}} u_{i}(x) \ell_{i}(x)$ is an EU representation of individual $i$ 's preferences. Since $u_{i}$ is determined up to (positive) affine transformations, we can assume it satisfies $u_{i}\left(x_{\min }\right)=v_{\min }$ and $u_{i}\left(x_{\max }\right)=v_{\max }$ (hence, $\varphi_{i}\left(v_{\min }\right)=v_{\min }$ and $\varphi_{i}\left(v_{\max }\right)=v_{\max }$, for all $\left.i\right)$.

Fact $4 \succcurlyeq$ can be represented by a separable function $\bar{V}(\gamma, \ell)=\sum_{i=1}^{n} \gamma_{i} \phi_{i}\left[U_{i}\left(\ell_{i}\right)\right]$. Choose $(\gamma, \ell) \in \Delta(I) \times \Delta(\mathscr{A})$, denote $v_{i}=\varphi_{i}\left(U_{i}\left(\ell_{i}\right)\right)$ and let $c_{i}\left(\ell_{i}\right) \in \mathscr{X}$ be individual $i$ 's certainty equivalent of the lottery $\ell_{i}$ (that is, $\left.u_{i}\left(c_{i}\left(\ell_{i}\right)\right)=U_{i}\left(\ell_{i}\right)\right)$. Consider a set of actions $\hat{A}=\left\{\hat{a}^{j} \mid j \in\{1, \ldots, n\}\right\}$ satisfying $\hat{a}^{1}(i)=c_{i}\left(\ell_{i}\right)$ and $\hat{a}^{j}(1)=\left(\varphi_{1} \circ u_{1}\right)^{-1}\left(v_{j}\right)$ for $i, j=1, \ldots, n$. By construction, $V\left(i, \hat{a}^{1}\right)=$ $\left(\varphi_{i} \circ u_{i}\right)\left(c_{i}\left(\ell_{i}\right)\right)=v_{i}$ and $V\left(1, \hat{a}^{i}\right)=\left(\varphi_{1} \circ u_{1}\right) \circ\left(\varphi_{1} \circ u_{1}\right)^{-1}\left(v_{i}\right)=v_{i}$. Hence, $\left(i, \hat{a}^{1}\right) \sim\left(1, \hat{a}^{i}\right)$ and, by source indifference and Lemma $2,\left(\gamma, \hat{a}^{1}\right) \sim\left(1, \ell^{\gamma}\right)\left(\ell^{\gamma}\right.$ is the action lottery on $\hat{A}$ associated with $\gamma)$. Put differently, $V\left(\gamma, \hat{a}^{1}\right)=V\left(1, \ell^{\gamma}\right)$. Note that by strong acceptance, $V(\gamma, \ell)=V\left(\gamma, \hat{a}^{1}\right)$. Therefore,

$$
\begin{aligned}
V(\gamma, \ell) & =V\left(\gamma, \hat{a}^{1}\right)=V\left(1, \ell^{\gamma}\right)=\varphi_{1}\left(U_{1}\left(\ell_{1}^{\gamma}\right)\right) \\
& =\varphi_{1}\left(\sum_{i=1}^{n} \gamma_{i} u_{1}\left(\left(\varphi_{1} \circ u_{1}\right)^{-1}\left(v_{i}\right)\right)\right) \\
& =\varphi_{1}\left(\sum_{i=1}^{n} \gamma_{i} \varphi_{1}^{-1}\left(v_{i}\right)\right) \\
& =\varphi_{1}\left(\sum_{i=1}^{n} \gamma_{i}\left(\varphi_{1}^{-1} \circ \varphi_{i}\right)\left(U_{i}\left(\ell_{i}\right)\right)\right)
\end{aligned}
$$

Denote $\bar{V}=\varphi_{1}^{-1} \circ V$ and $\phi_{i}=\varphi_{1}^{-1} \circ \varphi_{i}$ (note that $\bar{V}$ also represents the impartial observer preferences and its image is $\left.\left[v_{\min }, v_{\max }\right]\right)$. By the above,

$$
\bar{V}(\gamma, \ell)=\sum_{i=1}^{n} \gamma_{i} \phi_{i}\left[U_{i}\left(\ell_{i}\right)\right]
$$

Fact $5 \succcurlyeq$ can be represented by the affine function $\bar{V}(\gamma, \ell)=\sum_{i=1}^{n} \gamma_{i} U_{i}\left(\ell_{i}\right)$.

To conclude, we show that for all $i, \bar{V}_{i}=\phi_{i} \circ U_{i}$ is affine which, given $\varphi_{i}\left(v_{\min }\right)=v_{\min }$ and $\varphi_{i}\left(v_{\max }\right)=v_{\max }$, implies $\bar{V}_{i}=U_{i}$. Take $\ell, \ell^{\prime} \in \Delta(\mathscr{A})$. Since $U_{i}$ is of the EU type, we have for all $\lambda \in[0,1]$,

$$
\begin{aligned}
\bar{V}_{i}\left(\lambda \ell_{i}+(1-\lambda) \ell_{i}^{\prime}\right) & =\phi_{i}\left[U_{i}\left(\lambda \ell_{i}+(1-\lambda) \ell_{i}^{\prime}\right)\right] \\
& =\phi_{i}\left[\lambda U_{i}\left(\ell_{i}\right)+(1-\lambda) U_{i}\left(\ell_{i}^{\prime}\right)\right] \\
& =\phi_{i}\left[\lambda u_{i}\left(c_{i}\left(\ell_{i}\right)\right)+(1-\lambda) u_{i}\left(c_{i}\left(\ell_{i}^{\prime}\right)\right)\right] \\
& =\phi_{i}\left[U_{i}\left(\lambda c_{i}\left(\ell_{i}\right)+(1-\lambda) c_{i}\left(\ell_{i}^{\prime}\right)\right)\right]
\end{aligned}
$$




$$
\begin{aligned}
& =\bar{V}_{i}\left(\lambda \check{a}^{i}(i)+(1-\lambda) \check{a}^{j}(i)\right) \\
& =\bar{V}\left(i, \lambda \check{a}^{i}+(1-\lambda) \check{a}^{j}\right)
\end{aligned}
$$

for actions $\check{a}^{i}$ and $\check{a}^{j}$ satisfying $\check{a}^{i}(i)=c_{i}\left(\ell_{i}\right), \check{a}^{j}(i)=c_{i}\left(\ell_{i}^{\prime}\right)$ (note that the element $\lambda c_{i}\left(\ell_{i}\right)+(1-\lambda) c_{i}\left(\ell_{i}^{\prime}\right)$ that appears in the third line is a lottery, not an outcome). Defining $\check{a}^{i}(j)=\left(\phi_{j} \circ u_{j}\right)^{-1} \circ\left(\phi_{i} \circ u_{i}\right)\left(c_{i}\left(\ell_{i}^{\prime}\right)\right)$ we get

$$
\begin{aligned}
\bar{V}\left(j, \check{a}^{i}\right) & =\left(\phi_{j} \circ u_{j}\right) \circ\left(\phi_{j} \circ u_{j}\right)^{-1} \circ\left(\phi_{i} \circ u_{i}\right)\left(c_{i}\left(\ell_{i}^{\prime}\right)\right) \\
& =\left(\phi_{i} \circ u_{i}\right)\left(c_{i}\left(\ell_{i}^{\prime}\right)\right)=\bar{V}\left(i, \check{a}^{j}\right)
\end{aligned}
$$

and hence, by source indifference and for $\gamma$ satisfying $\gamma_{i}=\lambda, \gamma_{j}=1-\lambda$ and $\gamma_{k}=0$ otherwise,

$$
\bar{V}\left(i, \lambda \check{a}^{i}+(1-\lambda) \check{a}^{j}\right)=\bar{V}\left(\lambda i+(1-\lambda) j, \check{a}^{i}\right)
$$

(note that actions $\check{a}^{k}$ for $k \neq i, j$ are irrelevant but can easily be defined so as to fit with the requirements of the axiom). Now, by the structure of $\bar{V}$ and by using the equation $\bar{V}\left(j, \check{a}^{i}\right)=\bar{V}\left(i, \check{a}^{j}\right)$,

$$
\begin{aligned}
\bar{V}\left(\lambda i+(1-\lambda) j, \check{a}^{i}\right) & =\lambda \bar{V}\left(i, \check{a}^{i}\right)+(1-\lambda) \bar{V}\left(j, \check{a}^{i}\right) \\
& =\lambda \bar{V}\left(i, \check{a}^{i}\right)+(1-\lambda) \bar{V}\left(i, \check{a}^{j}\right) \\
& =\lambda \bar{V}_{i}\left(\check{a}^{i}(i)\right)+(1-\lambda) \bar{V}_{i}\left(\check{a}^{j}(i)\right) \\
& =\lambda \bar{V}_{i}\left(c_{i}\left(\ell_{i}\right)\right)+(1-\lambda) \bar{V}_{i}\left(c_{i}\left(\ell_{i}^{\prime}\right)\right) \\
& =\lambda \bar{V}_{i}\left(\ell_{i}\right)+(1-\lambda) \bar{V}_{i}\left(\ell_{i}^{\prime}\right)
\end{aligned}
$$

Summarizing (using (1)),

$$
\bar{V}_{i}\left(\lambda \ell_{i}+(1-\lambda) \ell_{i}^{\prime}\right)=\lambda \bar{V}_{i}\left(\ell_{i}\right)+(1-\lambda) \bar{V}_{i}\left(\ell_{i}^{\prime}\right)
$$

and the affinity of $\bar{V}_{i}$ is established.

Hence,

$$
\bar{V}(\gamma, \ell)=\sum_{i=1}^{n} \gamma_{i} U_{i}\left(\ell_{i}\right)
$$

as required.

\subsection{Preference for identity lotteries versus source indifference}

Lemma 3 If the impartial observer preferences satisfy strong acceptance, convexity and preference for identity lotteries, then they satisfy source indifference. 
Proof Consider, without loss of generality, a society $I=\{1, \ldots, n\}$, a set of available actions $A=\left\{a^{1}, \ldots, a^{n}\right\}$ and assume that (again, without loss of generality) $V\left(i, a^{1}\right)=V\left(1, a^{i}\right):=v_{i}$, for all $i$. Without loss of generality we can assume that all $v_{i}$ are pairwise different and that $v_{i}>v_{i+1}$ for all $i<n$. For $i, j \in\{1, \ldots, n\}$, let $x_{i j} \in \mathscr{X}$ be defined by $V_{i}\left(x_{i j}\right)=v_{\pi_{j}(i)}$, where $\pi_{j}$ is a permutation on $\{1, \ldots, n\}$ (as defined in the proof of the theorem), and note that, by the monotonicity of each $V_{i}$ with respect to the outcomes of $\mathscr{X}, V_{1}\left(x_{11}\right)>V_{1}\left(x_{12}\right)>\cdots>V_{1}\left(x_{1 n}\right)$, $V_{2}\left(x_{2 n}\right)>V_{2}\left(x_{21}\right)>V_{2}\left(x_{22}\right)>\cdots>V_{2}\left(x_{2(n-1)}\right), \ldots, V_{n}\left(x_{n 2}\right)>V_{n}\left(x_{n 3}\right)>$ $\cdots>V_{n}\left(x_{n n}\right)>V_{n}\left(x_{n 1}\right)$. Consider a new set of actions $\bar{A}=\left\{\bar{a}^{1}, \ldots, \bar{a}^{n}\right\}$ satisfying $\bar{a}^{j}(i)=x_{i j}$. By construction,

$$
V\left(i, \bar{a}^{1}\right)=V_{i}\left(x_{i 1}\right)=v_{\pi_{1}(i)}=v_{i}=V\left(i, a^{1}\right)
$$

and

$$
V\left(1, \bar{a}^{i}\right)=V_{1}\left(x_{1 i}\right)=v_{\pi_{i}(1)}=v_{i}=V\left(1, a^{i}\right)
$$

which implies that, by strong acceptance, $V\left(\gamma^{e}, a^{1}\right)=W\left(\gamma^{e},\left(v_{1}, \ldots, v_{n}\right)\right)=$ $V\left(\gamma^{e}, \bar{a}^{1}\right)$ and $V\left(1, \ell^{e}\right)$ given $A$ (where $\left.\ell^{e}=\sum_{j=1}^{n} \frac{1}{n} a^{j}\right)$ is equal to $V\left(1, \ell^{e}\right)$ given $\bar{A}$ (where $\ell^{e}=\sum_{j=1}^{n} \frac{1}{n} \bar{a}^{j}$ ). Hence, it is sufficient to restrict attention to $\bar{A}$ and to show that $V\left(\gamma^{e}, \bar{a}^{1}\right)=V\left(1, \ell^{e}\right)$ (given $\bar{A}$ ). For this note that: $(i)$ since $V\left(\gamma^{e}, \bar{a}^{i}\right)=W\left(\gamma^{e},\left(v_{1}, \ldots, v_{n}\right)\right)$ for all $i$, we have $V\left(\gamma^{e}, \bar{a}^{i}\right)=V\left(\gamma^{e}, \bar{a}^{j}\right)$, for all $i, j$; (ii) by construction, for every $k \in\{1, \ldots, n\}, V\left(i, \bar{a}^{k}\right)=V\left(k, \bar{a}^{i}\right)$, for all $i$; (iii) $V\left(\gamma^{e}, \ell^{e}\right) \in\left[\min _{i} V\left(i, \ell^{e}\right), \max _{i} V\left(i, \ell^{e}\right)\right]$ and hence, if $V\left(\gamma^{e}, \ell^{e}\right)=$ $\max _{i} V\left(i, \ell^{e}\right)$ then $V\left(\gamma^{e}, \ell^{e}\right)=V\left(i, \ell^{e}\right)=V\left(j, \ell^{e}\right)$, for all $i, j$; and $(i v)$ individual $i$ strictly prefers action $\bar{a}^{n+2-i}$ (where $\bar{a}^{n+2-1}=\bar{a}^{n+1}:=\bar{a}^{1}$ ) over all other actions and, by the monotonicity of $V_{i}$ with respect to first-order stochastic-dominance, he strictly prefers this action over all mixtures of the other actions. Therefore,

$$
V\left(\gamma^{e}, \bar{a}^{1}\right)=\max _{k} V\left(\gamma^{e}, \bar{a}^{k}\right) \geqslant \max _{k} V\left(k, \ell^{e}\right) \geqslant V\left(\gamma^{e}, \ell^{e}\right) \geqslant V\left(\gamma^{e}, \bar{a}^{1}\right)
$$

where the equality follows from $(i)$, the first inequality follows from (ii) and from preference for identity lotteries, the second inequality follows from the first part of (iii) and the last inequality follows from (iv) by repeated application of convexity (note that $\ell^{e}=\frac{1}{n} \sum_{j} \bar{a}^{j}$ ).

Since the first and the last elements are identical, $\max _{k} V\left(k, \ell^{e}\right)=V\left(\gamma^{e}, \ell^{e}\right)$ which, by the second part of (iii), implies that $V\left(1, \ell^{e}\right)=\max _{k} V\left(k, \ell^{e}\right)$ and, therefore, $V\left(1, \ell^{e}\right)=V\left(\gamma^{e}, \bar{a}^{1}\right)$. Hence, the impartial observer is indifferent between identity and action lotteries.

\subsection{GKPS's (2010) source indifference implies ours}

Lemma 4 Assume (as in GKPS 2010) that the impartial observer satisfies the following property: 
$\forall \gamma, \gamma^{\prime} \in \triangle(\mathscr{I}), \forall \ell, \ell^{\prime} \in \triangle(\mathscr{A})$ and $\forall \beta \in(0,1)$,

$$
\left(\gamma, \ell^{\prime}\right) \sim\left(\gamma^{\prime}, \ell\right) \Rightarrow\left(\beta \gamma+(1-\beta) \gamma^{\prime}, \ell\right) \sim\left(\gamma, \beta \ell+(1-\beta) \ell^{\prime}\right)
$$

Then, the impartial observer exhibits source indifference.

Proof The proof is by induction. Without loss of generality, consider a society $I=$ $\{1, \ldots, n\}$, the set of available actions $A=\left\{a^{1}, \ldots, a^{n}\right\}$ and assume that $\left(1, a^{i}\right) \sim$ $\left(i, a^{1}\right)$, for all $i$.

First let $n=2$. By the GKPS condition, $\left(1, a^{2}\right) \sim\left(2, a^{1}\right)$ implies

$$
\left(\frac{1}{2} 1+\frac{1}{2} 2, a^{1}\right) \sim\left(1, \frac{1}{2} a^{1}+\frac{1}{2} a^{2}\right)
$$

as required.

Next assume it holds for $n-1$ and consider $n$. Assume, without loss of generality, that the acts of $A$ satisfy $\left(i, a^{j}\right) \sim\left(i+1, a^{j-1}\right)$ for all $i \in\{1, \ldots, n-1\}, j \in$ $\{2, \ldots, n\}$. Consider the society $I^{\backslash 1}=\{2, \ldots, n\}$ and the set of actions $A^{\backslash n}=$ $\left\{a^{1}, \ldots, a^{n-1}\right\}$. By construction, $\left(2, a^{i}\right) \sim\left(i+1, a^{1}\right)$ for all $i=1, \ldots, n-1$ and hence, by the induction hypothesis, $\left(\frac{1}{n-1} \sum_{i=2}^{n} i, a^{1}\right) \sim\left(2, \frac{1}{n-1} \sum_{i=1}^{n-1} a^{i}\right)$. Next apply the same argument to $I^{\backslash n}=\{1, \ldots, n-1\}$ and $A^{\backslash n}=\left\{a^{1}, \ldots, a^{n-1}\right\}$, where $\left(2, a^{i}\right) \sim\left(i, a^{2}\right)$ for all $i$, to get $\left(2, \frac{1}{n-1} \sum_{i=1}^{n-1} a^{i}\right) \sim\left(\frac{1}{n-1} \sum_{i=1}^{n-1} i, a^{2}\right)$. Finally, apply it to $I^{\backslash n}=\{1, \ldots, n-1\}$ and $A^{\backslash 1}=\left\{a^{2}, \ldots, a^{n}\right\}$, where $\left(1, a^{i+1}\right) \sim\left(i, a^{2}\right)$ for all $i$, to get $\left(\frac{1}{n-1} \sum_{i=1}^{n-1} i, a^{2}\right) \sim\left(1, \frac{1}{n-1} \sum_{i=2}^{n} a^{i}\right)$. By transitivity,

$$
\left(\frac{1}{n-1} \sum_{i=2}^{n} i, a^{1}\right) \sim\left(1, \frac{1}{n-1} \sum_{i=2}^{n} a^{i}\right)
$$

To conclude, mix both sides of the last indifference with $\left(1, a^{1}\right)$ and, by the GKPS. condition, obtain $\left(\gamma^{e}, a^{1}\right) \sim\left(1, \ell^{e}\right)$ for $I=\{1, \ldots, n\}$ and $A=\left\{a^{1}, \ldots, a^{n}\right\}$, as required.

Open Access This article is distributed under the terms of the Creative Commons Attribution 4.0 International License (http://creativecommons.org/licenses/by/4.0/), which permits unrestricted use, distribution, and reproduction in any medium, provided you give appropriate credit to the original author(s) and the source, provide a link to the Creative Commons license, and indicate if changes were made.

\section{References}

Asheim, G.B., Mitra, T., Tungodden, B.: Sustainable recursive social welfare functions. Econ. Theory 49, 267-292 (2012)

Blackorby, C., Donaldson, D., Mongin, P.: Social aggregation without the expected utility hypothesis. Discussion Paper 2004-020, Ecole Polytechnique (2004)

Chew, S.H.: A generalization of the quasilinear mean with applications to the measurement of income inequality and decision theory resolving the Allais paradox. Econometrica 51, 1065-1092 (1983) 
Chew, S.H.: Axiomatic utility theories with the betweenness property. Ann. Oper. Res. 19, 273-298 (1989)

Chew, S.H., Karni, E., Safra, Z.: Risk aversion in the theory of expected utility with rank dependent probabilities. J. Econ. Theory 42, 370-381 (1987)

Dekel, E.: An axiomatic characterization of preferences under uncertainty: weakening the independence axiom. J. Econ. Theory 40, 304-318 (1986)

Dekel, E., Safra, Z., Segal, U.: Existence and dynamic consistency of Nash equilibrium with non-expected utility preferences. J. Econ. Theory 55, 229-246 (1991)

Dhillon, A., Mertens, J.F.: Relative utilitarianism. Econometrica 67, 471-498 (1999)

Diamond, P.A.: Cardinal welfare, individualistic ethics, and interpersonal comparison of utility: comment. J. Polit. Econ. 75, 765-766 (1967)

Elster, J.: Solomonic Judgements: Studies in the Limitation of Rationality. Cambridge University Press, Cambridge (1989)

Epstein, L., Segal, U.: Quadratic social welfare functions. J. Polit. Econ. 100, 691-712 (1992)

Ergin, H., Gul, F.: A theory of subjective compound lotteries. J. Econ. Theory 144, 899-929 (2009)

Fleurbaey, M., Mongin, P.: The utilitarian relevance of the aggregation theorem. Am. Econ. J. Microecon. 8, 289-306 (2016)

Gilboa, I., Samet, D., Schmeidler, D.: Utilitarian aggregation of beliefs and tastes. J. Polit. Econ. 112, 932-938 (2004)

Gorman, W.M.: The structure of utility functions. Rev. Econ. Stud. 35, 367-390 (1968)

Grant, S., Kajii, A., Polak, B., Safra, Z.: Generalized utilitarianism and Harsanyi's impartial observer theorem. Econometrica 78, 1939-1971 (2010)

Grant, S., Kajii, A., Polak, B., Safra, Z.: A generalized representation theorem for Harsanyi's ('impartial') observer. Soc. Choice Welf. 39, 833-846 (2012)

Harsanyi, J.C.: Cardinal utility in welfare economics and in the theory of risk-taking. J. Polit. Econ. 61, 434-435 (1953)

Harsanyi, J.C.: Cardinal welfare, individualistic ethics, and interpersonal comparisons of utility. J. Polit. Econ. 63, 309-321 (1955)

Harsanyi, J.C.: Nonlinear social welfare functions. Theory Decis. 6, 311-332 (1975)

Harsanyi, J.C.: Rational Behavior and Bargaining Equilibrium in Games and Social Situations. Cambridge University Press, Cambridge (1977)

Herstein, I.N., Milnor, J.: An axiomatic approach to measurable utility. Econometrica 21, 291-297 (1953)

Karni, E.: Impartiality: definition and representation. Econometrica 66, 1405-1415 (1998)

Karni, E., Safra, Z.: An extension of a theorem of von Neumann and Morgenstern with an application to social choice theory. J. Math. Econ. 34, 315-327 (2000)

Klaus, B., Klijn, F.: Procedurally fair and stable matching. Econ. Theory 27, 431-447 (2006)

Mongin, P., Pivato, M.: Ranking multidimensional alternatives and uncertain prospects. J. Econ. Theory 157, 146-171 (2015)

Quiggin, J.: A theory of anticipated utility. J. Econ. Behav. Organ. 3, 323-343 (1982)

Quiggin, J.: Generalized Expected Utility Theory: The Rank-Dependent Model. Kluwer, London (1993)

Segal, U.: Let's agree that all dictatorships are equally bad. J. Polit. Econ. 108, 569-589 (2000)

Sen, A.K.: Welfare inequalities and Rawlsian axiomatics. Theory Decis. 7, 243-262 (1976)

Sen, A.K.: Non-linear social welfare functions: a reply to Professor Harsanyi. In: Butts, R., Hintikka, J. (eds.) Foundational Problems in the Special Sciences, pp. 297-302. Reidel Publishing Company, Dordrecht (1977)

Weymark, J.A.: Generalized Gini inequality indices. Math. Soc. Sci. 1, 409-430 (1981)

Weymark, J.A.: A reconsideration of the Harsanyi-Sen debate on utilitarianism. In: Elster, J., Roemer, J.E. (eds.) Interpersonal Comparisons of Well-being, pp. 255-320. Cambridge University Press, Cambridge (1991)

Zhou, L.: Harsanyi’s utilitarianism theorems: general societies. J. Econ. Theory 72, 198-207 (1997) 